\title{
Regular consumption of a cocoa product improves the cardiometabolic profile in healthy and moderately hypercholesterolaemic adults
}

\author{
Beatriz Sarriá ${ }^{1 *} \dagger$, Sara Martínez-López ${ }^{1} \dagger$, José Luis Sierra-Cinos ${ }^{2}$, Luis García-Diz ${ }^{2}$, \\ Raquel Mateos ${ }^{1}$ and Laura Bravo ${ }^{1}$ \\ ${ }^{1}$ Department of Metabolism and Nutrition, Institute of Food Science, Technology and Nutrition (ICTAN-CSIC), \\ José Antonio Nováis 10, 28040 Madrid, Spain \\ ${ }^{2}$ Department of Nutrition and Bromatology I, Pharmacy Faculty, Complutense University of Madrid (UCM), \\ Ciudad Universitaria s/n, 28040 Madrid, Spain
}

(Submitted 15 January 2013 - Final revision received 8 May 2013 - Accepted 27 May 2013 - First published online 4 July 2013)

\begin{abstract}
Cocoa products present great health potential due to their high content of polyphenols, mainly of flavanols. However, the antioxidant, anti-inflammatory and other health effects of regularly consuming cocoa products seem to depend on the intake and health status of the consumer, etc. and need to be further clarified. A randomised, controlled, cross-over, free-living study was carried out in healthy ( $n$ 24) and moderately hypercholesterolaemic ( $>2000 \mathrm{mg} / 1, n 20)$ subjects to assess the influence of regularly consuming (4 weeks) two servings ( $15 \mathrm{~g}$ each) of a cocoa product rich in fibre (containing $33.9 \%$ of total dietary fibre (TDF) and $13.9 \mathrm{mg} / \mathrm{g}$ of soluble polyphenols) in milk $v$. consuming only milk (control) on (1) serum lipid and lipoprotein profile, (2) serum malondialdehyde levels, carbonyl groups, ferric reducing/antioxidant power, oxygen radical absorbance capacity and free radical-scavenging capacity, (3) IL- $1 \beta$, IL-6, TNF- $\alpha$, IL-10, IL-8, monocyte chemoattractant protein-1, and vascular and intracellular cell adhesion molecule levels, and (4) systolic and diastolic blood pressure and heart rate. Throughout the study, the diet and physical activity of the volunteers, as well as any possible changes in weight or other anthropometric parameters, were also evaluated. The intake of TDF increased $(P<0 \cdot 001)$ to the recommended levels. Serum HDL-cholesterol (HDL-C) levels were increased $(P<0 \cdot 001)$, whereas glucose $(P=0 \cdot 029)$, IL-1 $\beta(P=0 \cdot 001)$ and IL-10 $(P=0 \cdot 001)$ levels were decreased. The rest of the studied cardiovascular parameters, as well as the anthropometric ones, remained similar. In conclusion, regularly consuming a cocoa product with milk improves cardiovascular health by increasing HDL-C levels and inducing hypoglycaemic and anti-inflammatory effects in healthy and hypercholesterolaemic individuals without causing weight gain.
\end{abstract}

Key words: Dietary fibre: Cocoa polyphenols: HDL-cholesterol: Cytokines: Adhesion molecules: Antioxidants: Inflammation

The potential health-promoting effects of cocoa products have gained extensive attention in the last few years. Most of these effects are attributed to the polyphenolic fraction of cocoa, especially to the flavonoid group of polyphenols, which in cocoa are mainly flavanols. In fact, cocoa has been defined as a functional food due to its high flavanol content ${ }^{(1)}$. Moreover, soluble cocoa products are also a source of dietary fibre (DF). Cocoa powder contains $1.75 \%$ of DF, whereas in chocolate production DF is largely discarded ${ }^{(2)}$. Nowadays, new soluble cocoa products enriched with dietary components such as DF and polyphenols are being introduced into the food market, and their beneficial health properties need to be assessed.

The cardio-protective properties of cocoa products in humans have been mainly attributed to their polyphenol content. Cocoa flavanols may decrease LDL oxidation, improve plasma antioxidant status, increase the levels of HDLcholesterol (HDL-C), decrease the levels of biomarkers of lipid oxidation and improve endothelial function by increasing vascular NO synthase activity; the effect of NO synthase has been observed in studies using endothelial cells and in isolated rabbit aortic rings ${ }^{(3)}$. Cocoa flavanols have been

\footnotetext{
Abbreviations: C + M, cocoa + milk intervention stage; CRP, C-reactive protein; DF, dietary fibre; HDL-C, HDL-cholesterol; ICAM-1, intracellular cell adhesion molecule 1; IDF, insoluble dietary fibre; M, milk intervention stage; MDA, malondialdehyde; NS, neutral sugars; SDF, soluble dietary fibre; SEQC, Sociedad Española de Bioquímica Clínica y Patología Molecular; TDF, total dietary fibre; UA, uronic acids; VCAM-1, vascular cell adhesion molecule 1 .
}

*Corresponding author: B. Sarriá, fax +34915493627 , email beasarria@ictan.csic.es

† Both authors contributed equally to this work. 
the subject of health claims: maintenance of normal blood pressure and protection of lipids from oxidative damage ${ }^{(4)}$. The European Food Safety Authority has recently ${ }^{(5)}$ issued a positive opinion on cocoa flavanols and maintenance of flow-mediated vasodilation, which evaluates the capacity of conduit artery, typically the brachial artery, to respond to an increase in blood flow by dilating, as consistent acute and chronic benefits of chocolate or cocoa on flow-mediated vasodilation have been described regardless of the dose consumed $^{(6,7)}$. Ried et al. ${ }^{(8)}$ concluded in their review that flavanol-rich chocolate and cocoa products may have a small but statistically significant effect in lowering blood pressure by $2-3 \mathrm{mmHg}$ in the short term. However, in 2010, the European Food Safety Authority ${ }^{(4)}$ Panel concluded that a cause-and-effect relationship could not be established between the consumption of cocoa flavanols and protection of lipids from oxidative damage based on the review by Ding et $a l .{ }^{(9)}$. In the review, two long-term studies have reported no effects on $\mathrm{F}_{2}$-isoprostanes ${ }^{(10,11)}$ and another has reported that plasma oxidised LDL concentrations were decreased in the low-, middle- and high-cocoa groups compared with baseline; however, changes between the intervention groups and the placebo group were not assessed ${ }^{(12)}$. Therefore, the antioxidant effects of regularly consuming cocoa products need further clarification. Recently, it has been shown that polyphenols contained in the water-insoluble cocoa fraction exhibit antioxidant actions in the gastrointestinal tract despite being bound to macromolecules using an in vitro digestion model $^{(13)}$. In acute studies, it has been shown that the consumption of a single dose of dark chocolate $(40 \mathrm{~g}$ of $74 \%$ cocoa) markedly improves endothelium-mediated flow-mediated vasodilation and plasma antioxidant status in young healthy smokers ${ }^{(14)}$ and that the intake of flavonoidrich dark chocolate $(40 \mathrm{~g})$ compared with that of cocoa-free control chocolate reduces plasma isoprostane levels in cardiac transplant recipients ${ }^{(15)}$.

Furthermore, the natural content of cocoa in $\mathrm{DF}^{(16)}$ may contribute to the beneficial cardiovascular effects, as it has been proposed that a protective effect of DF against CVD is mediated through direct or indirect effects on serum lipids, possibly through the up-regulation of HDL-C levels ${ }^{(17,18)}$. Although the health benefits of DF have been mainly attributed to soluble dietary fibre (SDF), insoluble dietary fibre (IDF) through the dilution of gastrointestinal contents may hinder the digestion and absorption of dietary fats ${ }^{(19)}$. In fact, our group observed that a cocoa product rich in IDF induced slight hypocholesterolaemic and marked hypotriacylglycerolaemic effects, also reducing malondialdehyde (MDA) levels in serum and in the liver of hyperlipidaemic rats without inducing changes in serum total antioxidant capacity ${ }^{(19)}$. When the same fibre-rich cocoa product was regularly consumed as part of a non-restrictive, Mediterranean-Spanish diet, blood pressure and serum glucose and MDA levels were decreased in moderately hypercholesterolaemic subjects, without affecting body weight ${ }^{(20)}$. Taking these beneficial cardiovascular results into account, a new cocoa product rich in insoluble fibre has been developed and the effects of its regular consumption on cardiovascular risk factors have been assessed in human subjects.

The influence of cocoa on inflammatory parameters seems to depend on the amount of cocoa consumed and the health status of the consumer. Regular consumption of one serving of dark chocolate for $3 \mathrm{~d}$ significantly decreases the low-grade inflammation biomarker C-reactive protein (CRP) levels, whereas with higher intakes this effect is not maintained $^{(21)}$. Hypercholesterolaemic postmenopausal women who consumed a high-flavanol cocoa beverage for 6 weeks had significantly lower levels of soluble vascular cell adhesion molecule 1 (VCAM-1) than those who consumed a lowflavanol cocoa beverage ${ }^{(22)}$. In contrast, the consumption of moderate amounts of cocoa-related products induced platelet-inhibiting effects, although the levels of inflammation biomarkers did not change ${ }^{(23)}$. DF has also been reported to exert anti-inflammatory effects. In postmenopausal women, a greater intake of total, soluble and insoluble DF was inversely associated with IL-6 and TNF- $\alpha$ receptor 2 (TNF- $\alpha-\mathrm{R} 2$ ) levels in plasma, but not with CRP levels ${ }^{(24)}$. In contrast, in women with type 2 diabetes, the highest intake of whole grains was associated with both lower CRP and TNF- $\alpha$ levels compared with the lowest intake of whole grains ${ }^{(25)}$.

In conclusion, intermediate and long, well-controlled, human studies that evaluate the effects of realistic, moderate consumption of cocoa products on cardiovascular health are necessary. Bearing this in mind, we conducted a study in healthy and hypercholesterolaemic subjects to examine the influence of regularly consuming for 4 weeks a commercially available cocoa product rich in fibre in milk $v$. consuming only milk (control) on a broad range of cardiovascular biomarkers: serum lipid and lipoprotein levels; serum oxidation and antioxidant biomarker levels; plasma inflammation biomarker levels; blood pressure; heart rate. As cocoa products are relatively high-energy foods, their addition to a diet may lead to weight gain, which is an undesirable confounding factor that may affect the studied parameters; therefore, a wide range of anthropometric parameters were controlled, as well as physical activity, which is a well-known protective factor against the occurrence and progression of CVD.

\section{Experimental methods}

\section{Subjects}

The present study was conducted according to the guidelines laid down in the Declaration of Helsinki, and all procedures were approved by the Clinical Research Ethics Committee of Hospital Universitario Puerta de Hierro Majadahonda in Madrid (Spain). Written informed consent was obtained from all subjects. Volunteer recruitment was carried out by placing advertisements in the Universidad Complutense campus as well as by giving short talks between lectures. The inclusion criteria were as follows: having total cholesterol concentrations $<2000$ and $>2000 \mathrm{mg} / 1$ for the normocholesterolaemic and hypercholesterolaemic groups, respectively; being non-vegetarian and non-smoker for both men and women and being non-pregnant for women; aged between 
18 and 55 years; not suffering from any chronic pathology or gastrointestinal disorder. None of the subjects had taken dietary supplements, laxatives or antibiotics 6 months before the start of the study. Their BMI was less than $30 \mathrm{~kg} / \mathrm{m}^{2}$.

On the whole, fifty subjects initially accepted to participate in the study and gave informed written consent; however, only forty-four completed it.

\section{Study design}

This was a randomised, controlled, cross-over, free-living study. After a 2-week run-in stage, in which consumption of the fruit, vegetables and beverages mentioned below was restricted, the subjects consumed two sachets of soluble cocoa powder per $d$, one for breakfast and the other as a snack between lunch and dinner (in order to reduce interferences with other food items) in $200 \mathrm{ml}$ of semi-skimmed milk (total intake: $400 \mathrm{ml} / \mathrm{d}$ in the cocoa + milk $(\mathrm{C}+\mathrm{M})$ intervention stage) or two $200 \mathrm{ml}$ servings of semi-skimmed milk for 4 weeks ( $400 \mathrm{ml} / \mathrm{d}$ in the milk (M) intervention stage) in a random order. Blood samples and blood pressure, heart rate and anthropometric measurements were taken at baseline and at the end of each intervention. The trial was conducted during autumn months. The soluble cocoa product was provided by Nutrexpa S.L. It contained $33.9 \%$ of total dietary fibre (TDF) and $13.88 \mathrm{mg} / \mathrm{g}$ of soluble polyphenols; therefore, the two servings of cocoa provided $10 \cdot 17 \mathrm{~g} / \mathrm{d}$ of TDF and $416.4 \mathrm{mg} / \mathrm{d}$ of polyphenols. The servings used $(15 \mathrm{~g} / \mathrm{sachet})$ in the present study corresponded to the quantity of the product that can reasonably be expected to be consumed. During the run-in stage and the intervention periods, consumption of other cocoa products, oranges, mandarins, apples, grapes, strawberries, berries in general, beets and onion, as well as their derived beverages, including wine and juices, and tea was restricted in order to reduce inter-individual differences in the intakes of DF and polyphenols.

\section{Dietary control and compliance}

The subjects were asked to maintain the same dietary habits throughout the study. Their dietary intake was regularly evaluated to control any possible changes. The volunteers were instructed on how to fill the dietary records before starting the study. In the run-in stage and at the end of the two intervention periods, the volunteers were asked to complete a $72 \mathrm{~h}$ detailed food intake report, specifying the ingredients and amounts of food consumed, including serving weights (when possible) and household measurements. Compliance was controlled by counting the number of cocoa servings provided to the volunteers before and after the interventions, as well as by weekly calling the volunteers. In order to assess energy intake and dietary composition, the programme DIAL (Department of Nutrition and Bromatology I, Pharmacy Faculty, Complutense Universidad of Madrid (UCM), Spain) was used. The intake of polyphenols was estimated using the program www.phenol-explorer.eu taking into account the Folin-Ciocalteu method.

\section{Cocoa fibre and polyphenol analysis}

The TDF of the cocoa product was analysed in triplicate defatted samples following the Association of Official Analytical Chemists (AOAC) method modified in our laboratory ${ }^{(26)}$. Briefly, the cocoa samples were treated with heat-stable $\alpha$-amylase (A-3306; Sigma), protease (P-3910) and amyloglucosidase (A-9913), followed by centrifugation ( $15 \mathrm{~min}, 3000 \mathrm{~g}$ ) instead of filtration to separate the soluble and insoluble fractions obtained after the enzymatic hydrolysis of digestible compounds. Supernatants were quantitatively collected and pellets were washed twice with $10 \mathrm{ml}$ of distilled water and centrifuged, and all the supernatants were combined. These were transferred into dialysis tubes (12000-14000 molecular weight cut-off (MWCO), Dialysis Tubing Visking; Medicell International Limited) and dialysed against water for $48 \mathrm{~h}$ at $25^{\circ} \mathrm{C}$ (water flow 7 litres/ $\mathrm{h}$ in a 43 litre reservoir). Dialysates (SDF) were hydrolysed with $1 \mathrm{M}-\mathrm{H}_{2} \mathrm{SO}_{4}$ at $100^{\circ} \mathrm{C}$ for $90 \mathrm{~min}$ and NSP was determined in the hydrolysates. The residues obtained after enzymatic hydrolysis of the samples (IDF) were dried overnight at room temperature and hydrolysed with $12 \mathrm{M}-\mathrm{H}_{2} \mathrm{SO}_{4}$ for $1 \mathrm{~h}$ at $30^{\circ} \mathrm{C}$ and then diluted with $1 \mathrm{M}-\mathrm{H}_{2} \mathrm{SO}_{4}$ at $100^{\circ} \mathrm{C}$ for $90 \mathrm{~min}$ with shaking. After acid hydrolysis, the samples were centrifuged ( $15 \mathrm{~min}, 3000 \mathrm{~g}$ ), pellets were washed twice with distilled water, and the combined supernatants were collected for the determination of NSP. The residues were dried at $105^{\circ} \mathrm{C}$ overnight and gravimetrically quantified as Klason lignin.

Uronic acids (UA) in the hydrolysates from both SDF and IDF were quantified spectrophotometrically by the Scott ${ }^{(27)}$ method using galacturonic acid as a standard. Neutral sugars (NS) were analysed by spectrophotometry following the Southgate method ${ }^{(28)}$. TDF was calculated as IDF + SDF; IDF was calculated as NSP + Klason lignin and SDF as NSP $(\mathrm{NSP}=\mathrm{UA}+\mathrm{NS})$. Polyphenols were extracted following a procedure set up by our group ${ }^{(29)}$ and were analysed spectrophotometrically as total polyphenols using the FolinCiocalteu reagent and gallic acid as a standard, and the extracts were characterised by HPLC-diode array detection (DAD) using an Agilent 1200 series liquid chromatograph ${ }^{(30)}$.

\section{Blood samples}

Blood samples were drawn after an overnight fasting for $8-10 \mathrm{~h}$ at baseline and after the consumption of milk or milk + cocoa for 4 weeks. Serum (without an anticoagulant) and plasma (EDTA-coated tubes) were separated by centrifugation and frozen at $-80^{\circ} \mathrm{C}$ until analysis.

\section{Biochemical parameters}

The lipid profile of the serum samples was determined following reference methods or methods recommended by Sociedad Española de Bioquímica Clínica y Patología Molecular (SEQC) using the Roche Cobas Integra 400 plus analyser (Roche Diagnostics). The concentrations of uric acid, creatinine and glucose were determined according to standardised spectrophotometric techniques, and the concentration of CRP was determined using an automatised ultrasensible 
turbidimetric method (AU2700 Biochemistry analyser; Olympus). Reference ranges used for all the biochemical parameters were those indicated by SEQC.

\section{Inflammation biomarkers}

The concentrations of cytokines IL-1 $\beta$, IL-6, TNF- $\alpha$, IL-10 and IL-8 in the plasma samples were determined using the High Sensitivity Human Cytokine MILLIPLEX MAP kit (Millipore Corporation), and those of monocyte chemoattractant protein-1, VCAM-1 and intracellular cell adhesion molecule 1 (ICAM-1) were determined using the Human Cardiovascular disease MILLIPEX MAP kit on a Luminex equipment (Luminex100/200; Luminex Corporation). High- and low-concentration quality controls were used with all the biomarkers.

\section{Oxidation and antioxidant biomarkers}

The concentration of MDA was determined as that of its hydrazone by HPLC using dinitrophenylhydrazine for derivatisation ${ }^{(31)}$. Serum samples were analysed directly, and standard MDA was prepared by acidic hydrolysis of 1,1,3,3-tetraethoxypropane in $1 \%$ sulphuric acid. The concentrations are expressed as nmol MDA $/ \mathrm{mm}$ in serum. Protein oxidation of the serum samples was measured as carbonyl group content according to the method of Richert et al. ${ }^{(32)}$. Absorbance was measured at $360 \mathrm{~nm}$, and carbonyl content is expressed as $\mathrm{nmol} / \mathrm{mg}$ protein using an extinction coefficient of $22000 \mathrm{nmol} / 1$ per $\mathrm{cm}$. Protein content of the serum samples was determined using the Bradford reagent.

The antioxidant capacity of the fibre-rich cocoa powder was evaluated in the soluble polyphenol extract and serum samples using the ferric reducing/antioxidant power assay ${ }^{(33)}$. Free radical-scavenging capacity of the cocoa product and serum samples was also measured using the radical monocation 2,2'-azinobis-(3-ethylbenzothiazoline-6-sulfonic acid) (ABTS) discolouration method ${ }^{(34)}$ with some modifications. The oxygen radical absorbance capacity of the serum samples was determined according to the method of Huang et al. ${ }^{(35)}$. For the three parameters, Trolox was used as a standard, and results are expressed as $\mu$ mol of Trolox equivalents/g DM of the product and per $\mathrm{ml}$ of serum.

\section{Blood pressure parameters}

Blood pressure and heart rate were measured using an automatic arm sphygmomanometer (BS 150; Pic Indolor Diagnostic). At baseline and after 4 weeks, the volunteers were asked to rest on a chair before the cuff was placed on their left arm. After they were rested for $5 \mathrm{~min}$, a second reading was taken on the same arm. Readings were compared, and if not in agreement within $10-15 \mathrm{mmHg}$, a third reading was taken.

\section{Anthropometric measurements}

At baseline and the two intervention periods, the total body and trunk fat percentage of the volunteers was assessed from tetrapolar bioimpedance measurements using a Tanita segmental body composition analyser BC-418 MA (Tanita Corporation). The device had a weighing system, which was used to weigh the volunteers, and their height was determined using a Holtain precision mechanical stadiometer (Holtain Limited). With these data, BMI was calculated according to the following formula: weight $(\mathrm{kg}) /$ height $(\mathrm{m})^{2}$. Brachial, waist, abdominal, hip and thigh circumferences were measured using a SECA 203 flexible tape (SECA UK Ltd) (Table 6). Tricipital and subscapular skin folds were measured using an anthropometric calliper (Harpender Anthropometer; Holtain Limited). By means of these biometry data, body density $^{(36)}$ and the percentage of body fat ${ }^{(37)}$ were calculated.

\section{Physical activity}

The participants were asked to maintain their usual level of physical activity during the study. The volunteers filled out a questionnaire before the start of the study to report on their occupation and consequently the level of physical activity involved. At the beginning and end of the study, physical activity levels were calculated using an adapted version of the Minnesota Leisure Time Physical Activity Questionnaire by Martínez-González et $a l^{(38)}$. Total energy expenditure from leisure time was obtained by making the assumption that one metabolic equivalent is approximately $4 \cdot 2 \mathrm{~kJ} / \mathrm{min}$ $(1 \mathrm{kcal} / \mathrm{min})$ for a $70 \mathrm{~kg}$ man. Taking this into account and knowing the duration that the activities were performed per day, the data are expressed as $\mathrm{kJ} / \mathrm{d}$.

\section{Statistical analysis}

Taking total cholesterol as the main variable, a sample size of twenty-three subjects per group was calculated in order to obtain a statistical power of $80 \%$ such that the study will detect a treatment difference at the 0.05 significance level, if the true difference between the treatments is $60 \mathrm{mg} / \mathrm{l}$. This is based on the assumption that the within-patient standard deviation of the response variable is 10 .

Data are presented as means with their standard errors, unless specified otherwise. Before the statistical analysis, the normality of distribution and the homogeneity of variance were verified using the Kolmogorov-Smirnov and Levene tests, respectively. The general linear model of variance for repeated measures was used to assess differences in all the parameters studied in response to the interventions. To exclude the carry-over effect for the two periods, we compared the differences in the parameters obtained from the group that started with the $\mathrm{C}+\mathrm{M}$ intervention with those in the parameters obtained from the group that started with the $\mathrm{M}$ intervention. In order to assess the effects of belonging to the normocholesterolaemic or hypercholesterolaemic group, the group was considered as an inter-individual factor. Differences within either the normocholesterolaemic or hypercholesterolaemic group were studied using the Bonferroni test. Statistical significance was set at $P<0.05$. The statistical analysis was carried out using the SPSS statistical package (version 19.0; SPSS, Inc., IBM Company) and Statgraphics Centurion XVII (Stat Point Technologies, Inc.). 


\section{Results}

\section{Cocoa product fibre and polyphenol analysis}

The percentage of TDF and the content of the major constituents of the SDF and IDF fractions (NS, UA and Klason lignin) in the studied cocoa product were as follows: the percentage of the SDF fraction, which accounted for approximately $5 \%$ of the TDF, was 1.68 (sem 0.10) \% DM, being formed by NS (0.69 (SEM 0.04) \% DM) and UA (0.99 (SEM 0.09) \% DM). Quantitatively, IDF was the main DF component of cocoa, accounting for $95 \%$ of TDF. Close to $60 \%$ of the IDF fraction corresponded to NSP, which was made of NS (19.06 (SEM 1.60) $\%$ DM) and UA (1.26 (SEM 0.07) \% DM), with the remaining being Klason lignin (11.90 (sEm 0.28) \% DM). Soluble polyphenols in cocoa analysed spectrophotometrically were 13.88 (SEM 0.24) $\mathrm{mg} / \mathrm{g} \mathrm{DM}$ and attending to HPLC-DAD analysis ( $n$ 6) cocoa contained 1.47 (SEM $0 \cdot 2) \mathrm{mg} / \mathrm{g}$ DM of total flavanols, $0.31 \quad(\mathrm{SEM} \quad 0.08) \mathrm{mg} / \mathrm{g}$ DM of epicatechin, 0.60 (sem 0.11$) \mathrm{mg} / \mathrm{g}$ DM of catechin and 0.55 (sEM 0.04) mg/g DM of procyanidin $\mathrm{B} 2$.

\section{Subjects}

Of the fifty volunteers who were enrolled into the study, six withdrew due to personal, health or professional reasons. Of the remaining forty-four volunteers, twenty-four were women with an average age of 25.75 (SD 6.29) years and a BMI of $22.2(\mathrm{SD} 2.42) \mathrm{kg} / \mathrm{m}^{2}$, and twenty were men with an average age of 32 (SD 10.04) years and a BMI of $25 \cdot 15$ (SD 3.94$) \mathrm{kg} / \mathrm{m}^{2}$. The baseline characteristics of the forty-four subjects who completed the study are given in Table 1 and are separated into normocholesterolaemic ( $n$ 24) and moderately hypercholesterolaemic ( $n$ 20).

\section{Dietary control and compliance}

Based on the reports of the volunteers and the number of servings returned after the interventions, dietary compliance was high. The $72 \mathrm{~h}$ intake reports were pre-filtered to exclude those that were not reliable. We considered reports unreliable if estimations of daily energy intake were $70 \%$ below the lightactivity energy intake recommendations or $130 \%$ above the normal-activity energy intake recommendations for age and sex group ${ }^{(39)}$, as well as unjustified differences of over $4184 \mathrm{~kJ}$ ( $1000 \mathrm{kcal})$ between the baseline and final reports. The $72 \mathrm{~h}$ detailed food intake reports (Table 2) showed that DF $(P<0 \cdot 001)$ and protein $(P<0 \cdot 001)$ intakes were statistically higher after the $\mathrm{C}+\mathrm{M}$ intervention stage than after the other stage. In contrast, the intakes of energy, polyphenols, carbohydrates, lipids, cholesterol and fatty acids (monosaturated, polysaturated and saturated) increased without reaching the level of statistical significance. For all the indicated parameters, both the groups exhibited the same behaviour after the consumption of cocoa, and no significant cocoa $\times$ group interaction was observed.

\section{Biochemical parameters}

Biochemical results are given in Table 3. After the interventions, total cholesterol, LDL-cholesterol and TAG levels did not show statistically significant differences; in contrast, HDL-C levels were significantly increased $(P<0 \cdot 001)$. The concentrations of glucose $(P=0.029)$ and creatinine $(P=0.005)$ were significantly decreased. Alanine aminotransferase and aspartate aminotransferase enzyme levels were significantly increased $(P=0 \cdot 001$ and $0 \cdot 001$, respectively), although both the parameters remained within their respective SEQC reference ranges of normality $(0-41$ and $0-38 \mathrm{U} / 1)$. The levels of urea were significantly increased $(P=0.001)$ in contrast to those of creatinine, which were decreased $(P=0.005)$ after the cocoa intervention, with both the parameters remaining within their respective SEQC reference ranges (100-500 and $5 \cdot 0-13.0 \mathrm{mg} / 1)$.

\section{Inflammatory and adhesion molecule biomarkers}

The values of inflammatory and adhesion molecule levels showed a high variability (Table 4). The values of IL-1 $\beta$, IL-6 and IL- 8 levels observed were within the normal physiological range described by Kokkonen et al. ${ }^{(40)}$, whereas those of IL-10 levels were above the corresponding reference range $(2 \cdot 4-6.6 \mathrm{pg} / \mathrm{ml})$ and those of TNF- $\alpha$ levels were below the reference range $(14 \cdot 2-61.7 \mathrm{pg} / \mathrm{ml})$. The values of IL-1 $\beta$, IL-6, IL- 8 and TNF- $\alpha$ levels were slightly decreased at the end of the intervention, particularly in the hypercholesterolaemic group, without reaching the level of statistical significance,

Table 1. Baseline characteristics of the participants in the study (Mean values and standard deviations)

\begin{tabular}{|c|c|c|c|c|c|c|c|c|}
\hline & \multicolumn{4}{|c|}{ Normocholesterolaemic ( $n$ 24) } & \multicolumn{4}{|c|}{ Hypercholesterolaemic (n 20) } \\
\hline & \multicolumn{2}{|c|}{ Men $(n 11)$} & \multicolumn{2}{|c|}{ Women (n 13) } & \multicolumn{2}{|c|}{ Men $(n 9)$} & \multicolumn{2}{|c|}{ Women (n 11) } \\
\hline & Mean & SD & Mean & SD & Mean & SD & Mean & SD \\
\hline Age (years) & $28 \cdot 1$ & 7.9 & $26 \cdot 1$ & $6 \cdot 1$ & $35 \cdot 7$ & $11 \cdot 2$ & 25.4 & $6 \cdot 8$ \\
\hline Weight (kg) & $75 \cdot 2$ & $10 \cdot 6$ & $60 \cdot 3$ & $7 \cdot 7$ & $81 \cdot 0$ & 14.9 & $58 \cdot 8$ & 5.5 \\
\hline $\mathrm{BMI}\left(\mathrm{kg} / \mathrm{m}^{2}\right)$ & $24 \cdot 1$ & 3.6 & $22 \cdot 0$ & $2 \cdot 6$ & $26 \cdot 2$ & 4.2 & $22 \cdot 4$ & $2 \cdot 3$ \\
\hline Systolic BP (mmHg) & 117 & $10 \cdot 2$ & 115 & $9 \cdot 1$ & 122 & $15 \cdot 3$ & 118 & 10.4 \\
\hline Diastolic BP $(\mathrm{mmHg})$ & 71.8 & 8.0 & $72 \cdot 8$ & 8.4 & $79 \cdot 8$ & $10 \cdot 3$ & 73.9 & 8.4 \\
\hline Heart rate $(\mathrm{bpm})$ & $65 \cdot 1$ & $10 \cdot 1$ & $69 \cdot 1$ & $9 \cdot 8$ & $63 \cdot 8$ & 9.5 & $82 \cdot 2$ & $8 \cdot 2$ \\
\hline
\end{tabular}

$\mathrm{BP}$, blood pressure; bpm, beats per min. 
in contrast to that of IL-10 levels, which was significantly lower $(P=0 \cdot 001)$. The value of monocyte chemoattractant protein-1 levels was within the normal range $(32-147 \mathrm{ng} / \mathrm{ml})$ and did not vary after the interventions.

The value of VCAM-1 levels in the normocholesterolaemic group was close to the higher limit of the normal physiological range (46-166 ng/ml), and in the hypercholesterolaemic group, the value of the observed levels was above the upper limit. In contrast, the values of ICAM-1 levels in both groups were within the physiological range $(39-79 \mathrm{ng} / \mathrm{ml})^{(40)}$, with that of the hypercholesterolaemic group being near the upper limit. The consumption of cocoa led to a slight decrease in the value of ICAM-1 levels in the normocholesterolaemic group and in that of VCAM-1 levels in the hypercholesterolaemic group (Table 4).

\section{Blood pressure parameters}

Based on the systolic and diastolic blood pressure results (Table 5), the subjects were normotensive (systolic blood pressure $<140 \mathrm{mmHg}$ and diastolic blood pressure $\geq 80 \mathrm{mmHg}$ ) at the start and end of the interventions, since no changes were observed. All blood pressure values throughout the study were higher in the hypercholesterolaemic group than in the normocholesterolaemic group. No changes were observed regarding heart rate.

\section{Oxidation and antioxidant biomarkers}

The levels of MDA and carbonyl group biomarkers did not show statistical changes (Table 5).

The ferric reducing power in serum evaluated by the ferric reducing/antioxidant power method was slightly increased at the end of the intervention periods, without reaching the level of statistical significance. Similarly, oxygen radical absorbance capacity and ABTS values in serum showed no significant differences after the $\mathrm{M}$ and $\mathrm{C}+\mathrm{M}$ interventions (Table 5).

\section{Anthropometric measurements}

The consumption of cocoa did not induce an increase in body weight or changes in the other anthropometric parameters measured except for a significant decrease $(P<0 \cdot 001)$ in the tricipital skin folds (Table 6). However, the percentage of body fat calculated from the skin-fold data did not show statistical differences and nor did any other anthropometric parameters.

\section{Physical activity}

The ocupation of all the volunteers involved low physical activity levels; therefore, their energy expenditure was calculated by taking into account only leisure time, and no statistical differences were observed throughout the study. The normocholesterolaemic group showed a mean energy expenditure of 993.96 (SEM 92.43) at baseline, 922.93 (SEM 124.14) at the $M$ intervention stage and 833.60 (SEM 104.72) at the $\mathrm{C}+\mathrm{M}$ intervention stage. The hypercholesterolaemic 
group exhibited a mean energy expenditure of 742.36 (SEM 101.25) at baseline, 664.18 (SEM 135.99) at the M intervention stage and 631.63 (SEM 114.71) at the $\mathrm{C}+\mathrm{M}$ intervention stage.

\section{Discussion}

The present study shows that regular consumption of a soluble cocoa product rich in DF as part of a typical Mediterranean-Spanish diet increases the intake of DF to the recommended levels and improves cardiovascular health by increasing HDL-C levels and decreasing glucose, IL-1 $\beta$ and IL-10 levels without leading to weight gain or other anthropometric changes.

Soluble cocoa powder is widely consumed in many different countries including Spain ${ }^{(41)}$ by different population groups, particularly as part of breakfast or between main meals. The consumption rate of two cocoa beverages per day reproduces real conditions in the Spanish population and may be considered moderate (http://www.cacaoychoco late.com/consumoen.html). The servings used correspond to the quantity of the product that can reasonably be expected to be consumed. In fact, the optimal health effects of cocoa on coronary artery disease prevention have been associated with a moderate intake, while healthy outcomes disappear at a high intake ${ }^{(42)}$.

Three important confounding factors, diet, physical activity and weight gain, were controlled throughout the present study. Regarding diet, this was a study with free-living subjects and the only dietary modification introduced, apart from consuming the cocoa product, was the restriction of the consumption of other cocoa products and the fruit and vegetables mentioned previously. All the methods used to estimate food intake present limitations; nevertheless, in order to obtain the most accurate data from the $72 \mathrm{~h}$ dietary records, the volunteers were trained to fill them out and meetings were held to reinforce dietary instructions and assist the volunteers to complete the records as precisely as possible. The intake of polyphenols (Table 2) increased after the consumption of cocoa and milk in both the groups due to the phenolic content of cocoa, which is particularly rich in monomeric (epicatechin and catechin) and oligomeric (procyanidins) flavonols ${ }^{(15,43)}$, although the increase was not statistically significant probably owing to the restriction of the consumption of fruit and vegetables containing high amounts of antioxidant compounds.

The intake of carbohydrates in both the study groups at the beginning and end of the study (Table 2) was below the recommended range according to the study of Moreiras et $a l .{ }^{(39)}$. Contrarily, lipid and protein intake levels in the normocholesterolaemic group were within the recommended range at baseline, in contrast to those in the hypercholesterolaemic group, which were slightly above the respective recommended nutrient ranges. The levels of both macronutrients increased, although not significantly in the case of carbohydrates after regular intake of cocoa and milk, which may be explained by the sucrose (34.4\%) and DF (33.9\%) content in the cocoa product and protein in milk. In accordance, the values of serum urea levels were significantly higher $(P=0.001)$ after the consumption of cocoa compared with 
baseline values (Table 3), although they remained within the SEQC reference range $(10-50 \mathrm{mg} / \mathrm{ml})$, in contrast to those of serum creatinine levels, which were decreased $(P=0.005)$, but remained within the SEQC reference range $(5.0-13.0 \mathrm{mg} / \mathrm{l})$. There is no gold standard method for evaluating the intake of DF. However, the assessment of the intake of DF using dietary food records is a well-accepted alternative, particularly if the influence of smoking, alcohol intake, sex and education is considered ${ }^{(44)}$. These factors were controlled, as the volunteers who participated in the present study were non-smokers, consumed very low amounts of alcohol and had a similar medium-high education level. In both the groups, the intake of DF at baseline and after the $\mathrm{M}$ intervention was within the range estimated in the Spanish population $(16.3-18.4 \mathrm{~g} / \mathrm{d})^{(45)}$ and below that in European countries $(18.5 \mathrm{~g} / \mathrm{d})^{(46)}$. The consumption of cocoa significantly increased the intake of DF $(P<0 \cdot 001)$ and the recommended levels ( 25 and $28 \mathrm{~g} / \mathrm{d}$ in women and men, respectively) ${ }^{(47)}$ would have been reached had cocoa been included in the normal diet of the volunteers without any restrictions.

After the consumption of the cocoa product, no changes in the total lipid levels or in the intake of SFA were observed. In accordance with that study of Cooper et al. ${ }^{(41)}$, the fatty acid composition of cocoa had neutral effects on blood lipid and lipoprotein levels, except on HDL-C levels, which were significantly higher $(P=0.008)$. The HDL-C level-increasing effect of cocoa had already been described in $1994^{(48)}$ in healthy subjects who consumed a diet containing a moderate amount of milk chocolate ( $46 \cdot 2 \mathrm{~g} / \mathrm{d}) v$. a group who consumed an isoenergetic high-carbohydrate snack. Similarly, the HDL-C effect has recently been described in high-risk CVD patients who regularly consumed $40 \mathrm{~g}$ of cocoa powder with milk for 4 weeks $^{(49)}$, as well as in other studies performed in patients with hypercholesterolaemia ${ }^{(8)}$ and in healthy subjects ${ }^{(6)}$. Interestingly, according to the meta-analysis carried out by Hooper et $a l^{(50)}$, the beneficial effects on HDL-C levels are greater in longer-term trials. Different mechanisms have been postulated for the rise in HDL-C levels induced by cocoa polyphenols such as the increase in the expression and secretion of apoA1, the synthesis of apoA1 and apoA2, the efflux cholesterol promoter and ATP-binding cassette transporter A1, and the activity of phospholipid transfer proteins as well as decreases in the levels of cholesteryl ester transfer protein and the possible formation of micelles in the intestine, thus modifying fat absorption ${ }^{(49)}$. In addition, the DF present in cocoa more than likely contributed to the up-regulation of HDL-cholesterol levels in accordance with previous studies $^{(14,15)}$. To a certain extent, the present results are in contrast with those reported by Tokede et al. ${ }^{(51)}$, who concluded that the consumption of dark chocolate/cocoa products induces beneficial effects on LDL levels and neutral effects on TAG and HDL levels in short- and intermediate-term interventions, although dark chocolate seems to be a more effective matrix for delivering flavanols than cocoa beverages.

Other mechanisms may underlie the cardiovascular benefits of DF, such as the reduction of postprandial glucose concentrations, as fibre-rich foods can delay glucose absorption from the small intestine ${ }^{(52)}$ and improve insulin sensitivity ${ }^{(53)}$. 
In fact, long-term, prospective observational studies have suggested that diets containing larger quantities of whole grains and DF are associated with a reduced risk of type 2 diabetes $^{(54)}$. However, in the meta-analysis mentioned previously $^{(50)}$, significant reductions in fasting serum insulin concentrations were described after cocoa interventions, but no effects were observed on fasting glucose levels. In contrast, in the present study, serum glucose levels were significantly decreased $(P=0.038)$, which is in line with the hypoglycaemic results described in moderately hypercholesterolaemic subjects after the consumption of a fibre-rich cocoa product for 8 weeks ${ }^{(8)}$.

Inflammation and endothelial dysfunction are important contributors to the development of atherosclerosis. IL- 6 and TNF- $\alpha$ levels may be stronger predictors of incident cardiovascular events than CRP levels, as any dietary influence would first influence IL- 6 and TNF- $\alpha$ levels ${ }^{(24)}$. In the present study, no significant changes in the levels of pro-inflammatory cytokines were observed after the consumption of cocoa and milk, although they were slightly decreased, in contrast to that observed in in vitro studies that indicate that cocoa exerts anti-inflammatory effects via TNF- $\alpha^{(1)}$. IL-10 directly inhibits the release of inflammatory cytokines by monocytes and neutrophils and can dampen the inflammatory response by inhibiting Th1-cell cytokine production. The antiinflammatory and anti-atherogenic properties of IL-10 have been demonstrated using several models of atherosclerosis in mice. In human subjects, the expression of IL-10 has been demonstrated in both coronary arteries and atherosclerotic plaques, and higher serum levels of IL-10 have been shown in atherosclerosis patients compared with controls, suggesting that the levels of IL-10, as an anti-inflammatory molecule, may be elevated in response to the pro-inflammatory environment of atherosclerosis ${ }^{(55)}$. In agreement with this, in the present study, the value of IL-10 levels was higher in the hypercholesterolaemic subjects $v$, the normocholesterolaemic subjects. Surprisingly, the values of IL-10 levels were significantly decreased after the $\mathrm{C}+\mathrm{M}$ intervention in both the groups, being $50 \%$ lower than their respective baseline ranges. IL-10 is involved in the inflammatory response by the downregulation of the synthesis of other cytokines, including that of IL- $1 \beta^{(56)}$, which is in accordance with the statistically significant reduction of IL-1 $\beta$ levels $(P=0 \cdot 011)$ observed in the present study. In vitro studies have shown that smaller flavanol fractions (monomer through tetramers) induce an anti-inflammatory response by suppressing IL-1 $\beta$ mRNA expression and protein secretion ${ }^{(57)}$. Nevertheless, no changes were observed in IL-8 and monocyte chemoattractant protein-1 levels throughout the study.

Soluble adhesion molecules are early biomarkers of alterations in vascular function that indirectly indicate vascular inflammation and endothelial cell activation. Dissimilar effects of flavanol-rich foods on cell adhesion molecule levels have been described. A low-dose intake of white and red wine decreases the serum concentrations of ICAM-1; however, only red wine decreases the serum concentrations of VCAM-1 ${ }^{(58)}$. In contrast, black tea lowers soluble P-selectin levels without affecting soluble ICAM-1 and VCAM-1 levels ${ }^{(59)}$. 
Table 6. Effects of the consumption of the cocoa product rich in dietary fibre on anthropometric parameters

(Mean values with their standard errors)

\begin{tabular}{|c|c|c|c|c|c|c|c|c|c|c|c|c|c|c|}
\hline & \multicolumn{6}{|c|}{ Normocholesterolaemic ( $n$ 24) } & \multicolumn{6}{|c|}{ Hypercholesterolaemic (n 20) } & \multirow{3}{*}{$\begin{array}{c}\mathrm{C} \\
P^{*}\end{array}$} & \multirow{3}{*}{$\begin{array}{c}\mathrm{C} \times \text { group } \\
P^{*}\end{array}$} \\
\hline & \multicolumn{2}{|c|}{ Baseline } & \multicolumn{2}{|c|}{$M$ intervention } & \multicolumn{2}{|c|}{$\begin{array}{c}\mathrm{C}+\mathrm{M} \\
\text { intervention }\end{array}$} & \multicolumn{2}{|c|}{ Baseline } & \multicolumn{2}{|c|}{$\begin{array}{c}M \\
\text { intervention }\end{array}$} & \multicolumn{2}{|c|}{$\begin{array}{c}C+M \\
\text { intervention }\end{array}$} & & \\
\hline & Mean & SEM & Mean & SEM & Mean & SEM & Mean & SEM & Mean & SEM & Mean & SEM & & \\
\hline Body weight (kg) & $67 \cdot 0$ & 2.72 & $67 \cdot 0$ & 2.74 & $67 \cdot 1$ & 2.74 & $68 \cdot 6$ & 2.98 & 68.9 & 3.00 & $69 \cdot 2$ & 3.00 & NS & NS \\
\hline BMI $\left(\mathrm{kg} / \mathrm{m}^{2}\right)$ & $23 \cdot 0$ & 0.70 & $22 \cdot 8$ & 0.71 & 23.1 & 0.71 & $24 \cdot 1$ & 0.77 & $24 \cdot 2$ & 0.78 & $24 \cdot 3$ & 0.78 & NS & NS \\
\hline \multicolumn{15}{|l|}{ Skin folds (mm) } \\
\hline Tricipital & $18 \cdot 3$ & $1.33^{\mathrm{a}}$ & $16 \cdot 7$ & $1.38^{\mathrm{b}}$ & $17 \cdot 1$ & $1 \cdot 39^{b}$ & $21 \cdot 3$ & 1.46 & $19 \cdot 9$ & 1.52 & $20 \cdot 6$ & 1.50 & $<0.001$ & NS \\
\hline Subscapular & $13 \cdot 0$ & 1.05 & $12 \cdot 3$ & 1.06 & $12 \cdot 5$ & 0.98 & $16 \cdot 2$ & $1 \cdot 14$ & $16 \cdot 0$ & $1 \cdot 16$ & $16 \cdot 1$ & 1.07 & NS & NS \\
\hline \multicolumn{15}{|c|}{ Circumferences (cm) } \\
\hline Brachial & 28.5 & 0.70 & $28 \cdot 6$ & 0.80 & $28 \cdot 1$ & 0.72 & $29 \cdot 2$ & 0.76 & $28 \cdot 7$ & 0.87 & 28.9 & 0.80 & NS & NS \\
\hline Waist & $77 \cdot 6$ & $2 \cdot 41$ & $74 \cdot 1$ & $2 \cdot 22$ & 74.5 & $2 \cdot 28$ & 79.8 & $2 \cdot 64$ & $78 \cdot 4$ & 2.43 & 78.9 & 2.50 & NS & NS \\
\hline Abdominal & $85 \cdot 3$ & 1.73 & $84 \cdot 2$ & 1.89 & $85 \cdot 4$ & 1.79 & $89 \cdot 1$ & 1.89 & $89 \cdot 3$ & $2 \cdot 07$ & $89 \cdot 6$ & 1.96 & NS & NS \\
\hline Hip & $98 \cdot 3$ & 1.24 & 98.4 & 1.27 & $97 \cdot 3$ & 1.47 & 99.2 & 1.36 & $99 \cdot 1$ & 1.39 & 99.6 & 1.61 & NS & NS \\
\hline Thigh & 53.7 & 1.33 & 53.0 & 0.90 & $53 \cdot 1$ & 0.91 & 53.9 & 1.46 & 53.7 & 0.98 & 53.4 & 1.00 & NS & NS \\
\hline
\end{tabular}

$\mathrm{M}$, milk; C + M, cocoa + milk; C, cocoa.

${ }_{\mathrm{a}, \mathrm{b}}$ Mean values within a row with unlike superscript letters were significantly different within either the normocholesterolaemic or hypercholesterolaemic group according to the Bonferroni test.

${ }^{*} P$ values were assessed using the general linear model of variance for repeated measures.

Hypercholesterolaemic postmenopausal women who consumed a high-flavanol cocoa beverage ( $446 \mathrm{mg}$ of total flavanols) for 6 weeks had significantly lower levels of sVCAM-1 compared with those consuming the low-flavanol cocoa beverage ( $43 \mathrm{mg}$ of total flavanols) ${ }^{(22)}$, with epicatechin and certain B-type dimers, as well as their related metabolites, being the candidates for the effects of high-flavanol cocoa beverage, as they inhibit the activation of the oxidative stress-sensitive nuclear transcription factor NF- $\mathrm{BB}$, a known promoter of VCAM-1 expression ${ }^{(22)}$. In the present study, VCAM-1 levels were slightly decreased; this small effect can be greatly attributed to cocoa polyphenols and their metabolites, as other flavanol-rich foods had been restricted during the study. In the hypercholesterolaemic group, ICAM-1 levels remained similar in contrast to that observed in a previous study ${ }^{(60)}$ in high-risk CVD subjects in whom ICAM-1 levels were significantly decreased after consuming $40 \mathrm{~g}$ of cocoa powder with milk ( $495 \mathrm{mg}$ of total polyphenols). Once again, the lack of effects on ICAM-1 levels in the present study may be attributed to the flavanol-rich food restrictions.

Observational data on the relationship between cocoa intake and cardiovascular health suggest that the consumption of cocoa is associated with lower blood pressure. When subgrouping by epicatechin dose, greater effects were observed at doses $>50 \mathrm{mg} / \mathrm{d}^{(50)}$. Accordingly, regular consumption of two servings of the cocoa product rich in fibre provided $9.3 \mathrm{mg} / \mathrm{d}$ of epicatechin (M Gomez-Juaristi, unpublished results) and did not induce hypotensive effects in the normocholesterolaemic or hypercholesterolaemic group. In addition, the participants of the present study were normotensive at baseline, and thus the present results are in agreement with those reported by Ried et al. ${ }^{(61)}$, who observed a significant reduction of blood pressure in hypertensive subjects but not in normotensive subjects after the consumption of cocoa, and with those of a previous study carried out in our group in moderately hypercholesterolaemic subjects whose diastolic blood pressure was near the lower limit of the range of high blood pressure at baseline $^{(20)}$.

There was no significant effect on lipid and protein oxidation biomarker levels and on serum ferric reducing power, free radical-scavenging activity in vivo or oxygen radical absorbance capacity. As the contribution of fruit and vegetables to the antioxidant status of the volunteers had been reduced, it seems that plasma cocoa polyphenols did not reach a concentration high enough or were not in a chemical form to show antioxidant activity, which is in line with the results reported by Turner et al. ${ }^{(62)}$ and Rimbach et al. ${ }^{(63)}$ However, it should not be disregarded that the study was not powered to evaluate changes in antioxidant parameters and also that when chocolate is mixed with milk the absorption of antioxidants is reduced ${ }^{(64)}$

With respect to the remaining confounding factors, physical activity and weight gain, energy expenditure at leisure time was relatively high. The assumption that one metabolic equivalent is approximately $4.2 \mathrm{~kJ} / \mathrm{min}(1 \mathrm{kcal} / \mathrm{min})$ may have led to an overestimation of energy expenditure. Nevertheless, that the physical activity level was not different throughout the study makes the possible influence of this factor uniform. Accordingly, none of the anthropometric parameters measured, weight, BMI, subscapular skin folds, or the brachial, waist, abdominal, hip and thigh circumferences, showed changes (Tables 1 and 6). A significant decrease in the tricipital skin folds in both the groups was observed, but this result was not supported by the biometry data. Therefore, the results of the present study are in line with that of Buijsse et $a l .{ }^{(65)}$, who reported that energy intake is higher in dark chocolate consumers than in non-consumers; however, the BMI was lower in the former group, although we did not observe a decrease in BMI. This 'antiobesity' effect has been attributed to the physiological activity of polyphenols, which has been described in human subjects who consumed tea ${ }^{(66)}$ and rats that consumed $\operatorname{cocoa}^{(67)}$. In the latter study, the 
antiobesity effects of cocoa polyphenols were explained by the modulation of lipid metabolism, especially by decreasing fatty acid synthesis and transport system activity, and the enhancement of part of the thermogenesis mechanism in the liver and white adipose tissue.

The present study has limitations: the lack of blinding of subjects and investigators may have led to certain bias; the number of subjects was relatively small; treatment duration was relatively short to predict the effects of habitual cocoa intake on the cardiovascular parameters studied. Finally, it is not possible to specify to what extent the observed effects are due to DF, polyphenols or other ingredients of cocoa.

\section{Concluding remarks}

In recent years, there has been growing interest in cocoa and health. Although many studies have shown that cocoa induces cardioprotective effects, certain related aspects need to be clarified. This was the aim of the present study, in which the effects of regular consumption of a cocoa product rich in fibre $(33.9 \% \mathrm{TDF}$, providing $10.17 \mathrm{~g}$ of TDF and $416.4 \mathrm{mg}$ of polyphenols/d) within a typical Mediterranean-Spanish diet were assessed. According to the results obtained, it may be concluded that:

(1) cocoa is an efficacious alternative to increase the intake of DF to the recommended levels, without leading to weight gain or other anthropometric changes;

(2) it increases serum HDL-C levels, without affecting other lipid or lipoprotein parameters;

(3) it decreases plasma IL-1 $\beta$ and IL-10 concentrations and slightly lowers other pro-inflammatory molecule concentrations;

(4) it reduces fasting serum glucose levels without changing fasting serum antioxidant or blood pressure values;

(5) there are no differences in the effects observed between the moderately hypercholesterolaemic and normocholesterolaemic subjects.

In contrast to chocolate, where the long-term side effects and optimal dose need to be questioned, we conclude that regular and moderate consumption of cocoa might be a worthwhile dietary approach for improving cardiovascular health.

\section{Acknowledgements}

The authors thank the volunteers who participated in the present study, Laura Barrios for her statistical assistance and Aránzazu Fernández-Espinosa for her technical assistance. They also acknowledge Project Consolider-Ingenio (CSD 2007-00063) from the Spanish Ministry of Science and Innovation. S. M.-L. thanks the Spanish National Research Council for her predoctoral fellowship under the JAE-Pre programme funded by the European Social Fund. The present study was funded by Nutrexpa S.L. The authors' contributions were as follows: L. B. was involved in the trial conception, design and data interpretation; S. M.-L. and B. S. conducted the study and carried out the statistical analysis of data; S. M.-L., B. S. and R. M. carried out the assays and interpreted the results; J. L. S.-C. and L. G.-D. carried out the anthropometric measurements and statistically analysed the corresponding data; B. S. wrote the initial draft and all authors critically reviewed it and contributed to the final version of the manuscript. There are no conflicts of interest.

\section{References}

1. Selmi C, Cocchi CA, Lanfredini M, et al. (2008) Chocolate at heart: the anti-inflammatory impact of cocoa flavonoids. Mol Nutr Food Res 52, 1340-1348.

2. Jenkins DJA, Kendall CWC, Vuksan V, et al. (2000) Effect of cocoa bran on low-density lipoprotein oxidation and fecal bulking. Arch Intern Med 160, 2374-2379.

3. Rimbach G, Melchin M, Moehring J, et al. (2009) Polyphenols from cocoa and vascular health - a critical review. Int J Mol Sci 10, 4290-4309.

4. European Food Safety Agency (2010) Scientific Opinion on the substantiation of health claims related to cocoa flavanols and protection of lipids from oxidative damage (ID 652, 1506, 3143), and maintenance of normal blood pressure (ID 1507) pursuant to Article 13(1) of Regulation (EC) No. 1924/20061. EFSA J 8, 1792 [21 pp.].

5. European Food Safety Agency (2012) Scientific Opinion on the substantiation of a health claim related to cocoa flavanols and maintenance of normal endothelium-dependent vasodilation pursuant to Article 13(5) of Regulation (EC) No. 1924/ 20061. EFSA J 10, 2809 [21 pp.].

6. Davison K, Coates AM, Buckley JD, et al. (2008) Effect of cocoa flavanols and exercise on cardiometabolic risk factors in overweight and obese subjects. Inter J Obes 32 , 1289-1296.

7. Hooper L, Kay C, Abdelhamaid A, et al. (2012) Effects of chocolate, cocoa, and flavan-3-ols on cardiovascular health: a systematic review and meta-analysis of randomized trials. Am J Clin Nutr 95, 740-751.

8. Ried K, Sullivan TR, Fakler P, et al. (2012) Effect of cocoa on blood pressure. The Cochrane Database of Systematic Reviews, issue 8, CD008893.

9. Ding EL, Hutfless SM, Ding X, et al. (2006) Chocolate and prevention of cardiovascular disease: a systematic review. Nutr Metab 3, 2.

10. Mursu J, Voutilainen S, Nurmi T, et al. (2004) Dark chocolate consumption increases HDL cholesterol concentration and chocolate fatty acids may inhibit lipid peroxidation in healthy humans. Free Radic Biol Med 37, 1351-1359.

11. Mathur S, Devaraj S, Grundy SM, et al. (2002) Cocoa products decrease low density lipoprotein oxidative susceptibility but do not affect biomarkers of inflammation in humans. J Nutr 132, 3663-3667.

12. Baba S, Natsume M, Yasuda A, et al. (2007) Plasma LDL and HDL cholesterol and oxidized LDL concentrations are altered in normo- and hypercholesterolemic humans after intake of different levels of cocoa powder. J Nutr 137, 1436-1441.

13. Fogliano V, Corollaro ML, Vitaglione $\mathrm{P}$, et al. (2011) In vitro bioaccesibility and gut biotransformation of polyphenols present in the water-insoluble cocoa fraction. Mol Nutr Food Res 55, S44-S55.

14. Hermann F, Spieker LE, Ruschitzka F, et al. (2006) Dark chocolate improves endothelial and platelet function. Heart 92, 119-120.

15. Flammer AJ, Hermann F, Sudano I, et al. (2007) Dark chocolate improves coronary vasomotion and reduces platelet reactivity. Circulation 116, 2376-2382. 
16. Lecumberri E, Mateos R, Izquierdo-Pulido M, et al. (2007) Dietary fibre composition, antioxidant capacity and physico-chemical properties of a fibre-rich product from cocoa (Theobroma cacao L.). Food Chem 104, 948-954.

17. Wu H, Dwyer KM, Fan Z, et al. (2003) Dietary fiber and progression of atherosclerosis: the Los Angeles Atherosclerosis Study. Am J Clin Nutr 78, 1085-1091.

18. Tillotson JL, Grandits GA, Bartsch GE, et al. (1997) Relation of dietary fiber to blood lipids in the special intervention and usual care groups in the Multiple Risk Factor Intervention Trial. Am J Clin Nutr 65, 327S-337S.

19. Lecumberri E, Goya L, Mateos R, et al. (2007) A diet rich in dietary fiber from cocoa improves lipid profile and reduces malondialdehyde in hypercholesterolemic rats. Nutrition 23, 332-341.

20. Sarriá B, Mateos R, Sierra-Cinos JL, et al. (2012) Hypotensive, hypoglycaemic and antioxidant effects of consuming a cocoa product in moderately hypercholesterolemic humans. Food Funct 3, 867-874.

21. Di Giuseppe R, Castelnuovo AD, Centritto F, et al. (2008) Regular consumption of dark chocolate is associated with low serum concentrations of C-reactive protein in healthy Italian population. J Nutr 138, 1939-1945.

22. Wang-Polagruto JF, Villablanca AC, Polagruto JA, et al. (2006) Chronic consumption of flavanol-rich cocoa improves endothelial function and decreases vascular cell adhesion molecule in hypercholesterolemic postmenopausal women. Pharmacol 47, Suppl. 2, S177-S186.

23. Ostertag LM, O'Kennedy N, Kroon PA, et al. (2010) Impact of dietary polyphenols on human platelet function - a critical review of controlled dietary intervention studies. Mol Nutr Food Res 54, 60-81.

24. Ma Y, Hebert JR, Li W, et al. (2008) Association between dietary fiber and markers of systemic inflammation in the Women's Health Initiative Observational Study. Nutrition 24, 941-949.

25. Qi L, van Dam RM, Liu S, et al. (2006) Wholegrain, bran, and cereal fiber intakes and markers of systemic inflammation in diabetic women. Diabetes Care 29, 207-211.

26. Saura-Calixto F, García-Alonso A, Goñi I, et al. (2000) In vitro determination of the indigestible fraction in foods: an alternative to dietary fiber analysis. J Agric Food Chem $\mathbf{4 8}$, 3342-3347.

27. Scott RW (1979) Colorimetric determination of hexuronic acids in plant materials. Anal Chem 51, 936-941.

28. Southgate DAT (1976) Determination of Food Carbohydrates. London: Applied Science Publishers Ltd.

29. Bravo L \& Saura-Calixto F (1998) Characterization of the dietary fiber and the in vitro indigestible fraction of grape pomace. Am J Enol Viticult 49, 135-141.

30. Cienfuegos-Jovellanos E, Quiñones MM, Muguerza B, et al. (2009) Antihypertensive effect of a polyphenol-rich cocoa powder industrially processed to preserve the original flavonoids of the cocoa beans. J Agric Food Chem 57, 6156-6162.

31. Mateos R, Lecumberri E, Ramos S, et al. (2005) Determination of malondialdehyde (MDA) by high-performance liquid chromatography in serum and liver as a biomarker for oxidative stress: application to a rat model for hypercholesterolemia and evaluation of the effect of diets rich in phenolic antioxidants from fruits. J Chromatogr B Analyt Technol Biomed Life Sci 827, 76-82.

32. Richert S, Wehr NB, Stadtman ER, et al. (2002) Assessment of skin carbonyl content as a noninvasive measure of biological age. Arch Biochem Biophys 397, 430-432.
33. Pulido R, Bravo L \& Saura-Calixto F (2000) Antioxidant activity of dietary polyphenols as determined by a modified ferric reducing/antioxidant power assay. J Agric Food Chem 48, 3396-3402.

34. Re R, Pellegrini N, Proteggente A, et al. (1999) Antioxidant activity applying an improved ABTS radical cation decolorization assay. Free Radic Biol Med 26, 1231-1237.

35. Huang D, Ou B, Hampsch-Woodill M, et al. (2002) Highthroughput assay of oxygen radical absorbance capacity (ORAC) using a multichannel liquid handling system coupled with a microplate fluorescence reader in 96-well format. J Agric Food Chem 50, 4437-4444.

36. Siri WE (1961) Body composition from fluid spaces and density: analysis of methods. In Techniques for Measuring Body Composition, pp. 223-244 [J Brozeck and A Henschel, editors]. Washington, DC: National Academy of Sciences.

37. Durnin JVGA \& Womersley J (1974) Body fat assessed from total body density and its estimation from skinfold thickness: measurements on 481 men and women aged from 16 to 72 years. Br J Nutr 32, 77-97.

38. Martínez-González MA, López-Fontana C, Varo JJ, et al. (2005) Validation of the Spanish version of the physical activity questionnaire used in the Nurses' Health Study and the Health Professionals' Follow-up Study. Public Health Nutr 8, 920-927.

39. Moreiras O, Carbajal A, Cabrera L, et al. (2009) Tablas de composición de alimentos (Food Composition Tables), 13th ed. Madrid: Ediciones Pirámide S.A..

40. Kokkonen H, Söderstrom I, Rocklöv J, et al. (2010) Upregulation of cytokines and chemokines predates the onset of rheumatoid arthritis. Arthritis Rheum 62, 383-391.

41. Cooper KA, Donovan JL, Waterhouse AL, et al. (2008) Cocoa and health: a decade of research. Br J Nutr 99, 1-11.

42. Di Castelnuovo A, di Giuseppe R, Lacoviello L, et al. (2012) Consumption of cocoa, tea and coffee and risk of cardiovascular disease. Eur J Intern Med 23, 15-25.

43. Gómez-Juaristi M, González-Torres L, Bravo L, et al. (2011) Beneficial effects of chocolate on cardiovascular health. Nutr Hosp 26, 289-292.

44. Loening-Baucke V, Miele E \& Staiano A (2004) Fiber (glucomannan) is beneficial in the treatment of childhood constipation. Pediatrics 113, 259-264.

45. Saura-Calixto FD \& Goñi I (2004) The intake of dietary indigestible fraction in the Spanish diet shows the limitations of dietary fibre data for nutritional studies. Eur J Clin Nutr $\mathbf{5 8}$ $1078-1082$.

46. Tabernero M, Serrano J \& Saura-Calixto F (2007) Dietary fiber intake in two European diets with high (Copenhagen, Denmark) and low (Murcia, Spain) colorectal cancer incidence. J Agric Food Chem 55, 9443-9449.

47. Kassis AN, Santosa S \& Jones PJH (2009) Potential health claims on dietary fiber: how robust is the evidence? Int $J \mathrm{~N}$ M 4, 28-32.

48. Kris-Etherton PM \& Mustad VA (1994) Chocolate feeding studies: a novel approach for evaluating the plasma lipids effects of stearic acid. Am J Clin Nutr 60, 1029S-1036S.

49. Khan N, Monagas M, Andres-Lacueva C, et al. (2011) Regular consumption of cocoa powder with milk increases HDL cholesterol and reduces oxidized LDL levels in subjects at high-risk of cardiovascular disease. Nutr Metab Cardiovasc Dis 22, 1046-1053.

50. Hooper L, Kroon PA, Rimm EB, et al. (2008) Flavonoids, flavonoid-rich foods, and cardiovascular risk: a meta-analysis of randomized controlled trials. Am J Clin Nutr 88, 38-50. 
51. Tokede OA, Gaziano JM \& Djoussé L (2011) Effects of cocoa products/dark chocolate on serum lipids: a meta-analysis. Eur J Clin Nutr 65, 879-886.

52. Giacco R, Parillo M, Rivellese AA, et al. (2000) Longterm dietary treatment with increased amounts of fiber-rich low-glycemic index natural foods improves blood glucose control and reduces the number of hypoglycemic events in type 1 diabetic patients. Diabetes Care 23, 1461-1466.

53. van der Du DLA, Boshuizen HC, Forouhi NG, et al. (2009) Dietary fiber and subsequent changes in body weight and waist circumference in European men and women. $A m \mathrm{~J}$ Clin Nutr 91, 329-336.

54. Schulze M, Hoffmann K, Manson J, et al. (2005) Dietary pattern, inflammation, and incidence of type 2 diabetes in women. Am J Clin Nutr 82, 675-682.

55. Lakoski SG, Liu Y, Brosnihan KB, et al. (2008) Interleukin-10 concentration and coronary heart disease (CHD) event risk in the estrogen replacement and atherosclerosis (ERA) study. Atherosclerosis 197, 443-447.

56. Heiskanena M, Kähönen M, Hurme M, et al. (2010) Polymorphism in the IL10 promoter region and early markers of atherosclerosis: The Cardiovascular Risk in Young Finns Study. Atherosclerosis 208, 190-196.

57. Mao TK, Powell JJ, van de Water J, et al. (2000) The effect of cocoa procyanidins on the transcription and secretion of interleukin $1 \beta$ in peripheral blood mononuclear cells. Life Sci 66, 1377-1386.

58. Sacanella E, Vázquez-Agell M, Mena MP, et al. (2007) Downregulation of adhesion molecules and other inflammatory biomarkers after moderate wine consumption in healthy women: a randomized trial. Am J Clin Nutr 86, 1463-1469.
59. Hodgson JM, Puddey IB, Mori TA, et al. (2001) Effects of regular ingestion of black tea on haemostasis and cell adhesion molecules in humans. Eur J Clin Nutr $\mathbf{5 5}$, 881-886.

60. Monagas M, Khan N, Andrés-Lacueva C, et al. (2009) Effect of cocoa powder on the modulation of inflammatory biomarkers in patients at high risk of cardiovascular disease. Am J Clin Nutr 90, 1144-1150.

61. Ried K, Sullivan T, Fakler P, et al. (2010) Does chocolate reduce blood pressure? A meta-analysis. BMC Med 8, 39-49.

62. Turner R, Baron T, Wolffram S, et al. (2004) Effect of circulating forms of soy isoflavones on the oxidation of low density lipoprotein. Free Radic Res 38, 209-216.

63. Rimbach G, Weinberg PD, de Pascual-Teresa S, et al. (2004) Sulfation of genistein alters its antioxidant properties and its effect on platelet aggregation and monocyte and endothelial function. Biochim Biophys Acta 1670, 229-237.

64. Serafini M, Bugianesi R, Maiani G, et al. (2003) Plasma antioxidants from chocolate. Nature 424, 1013.

65. Buijsse B, Feskens EJM, Kok FJ, et al. (2006) Cocoa intake, blood pressure and cardiovascular mortality. Arch Intern Med 166, 411-417.

66. Nagao T, Komine $\mathrm{Y}$, Soga $\mathrm{S}$, et al. (2005) Ingestion of a tea rich in catechins leads to a reduction in body fat and malondialdehyde-modified LDL in men. Am J Clin Nutr 81, 122-129.

67. Matsui N, Itoa R, Nishimura E, et al. (2005) Ingested cocoa can prevent high-fat diet-induced obesity by regulating the expression of genes for fatty acid metabolism. Nutrition $\mathbf{2 1}$, 594-601. 